\title{
Measurement and Analysis of Learning Engagement of South-Asian Students in Chinese Universities 南亚国家来华留学生个体学习性投入的测量与分析
}

\author{
Lan Yu 余蓝 \\ Beijing Language and Culture University, China \\ 北京语言大学, 中国 \\ Shucheng Zhu 朱述承 \\ Beijing Language and Culture University, China \\ 北京语言大学, 中国
}

\begin{abstract}
International students' learning experiences and learning outcomes in intercultural contexts are important topics in higher education internationalization. This study focused on South-Asian students studying at Chinese universities. To assess the participants' academic engagement during their study at Chinese universities, the Individual South-Asian Student Engagement Questionnaire was developed. Through the use of exploratory factor analysis and correlation analysis on a sample of 193 SouthAsian students in China, the research confirmed the reliability and validity of the instrument. Four dimensions, i.e. learning motivation, learning behaviors, learning strategies and learning outcomes, as well as the interactions between the four dimensions, were suggested to understand the features of South-Asian students' learning engagement during their studies in Chinese higher education institutions.
\end{abstract}

摘要：国际学生在跨文化情境下的学习体验与收获是高等教育国际化研究领域的 重要议题。本研究选取具有区域性、国别化特征的南亚国家来华留学生为研究对 象, 通过实施来华留学生个体学习性投入调查收集数据, 采用统计学分析检验该 测量工具的信效度, 同时呈现南亚留学生在学习动机、学习行为、学习策略和学 业成就四个维度的关键特征及其相互关系。

Keywords: South-Asian students, individual student engagement, questionnaire 关键 词: 南亚国家来华留学生, 个体学习性投入, 测量工具 
南亚国家来华留学生人数近几年呈现显著的增长趋势, 既有传统的地缘优势和经 济交往使然，也有 “一带一路” 国际教育交流与合作的推动效应。在 2005-2014 年间, 南亚 8 国来华留学总人数从 3,295 人增加到 35,494 人, 增幅达到 $30.23 \%$ （陈丽 \& 艾孜买提, 2016）。2018 年, 巴基斯坦、印度首次超过美国而成为排 名仅次于韩国、泰国的来华留学生生源地国, 占当年来华留学总人数的比例分别 为 $5.69 \%$ 和 $4.71 \%$ (中华人民共和国教育部, 2019)。本研究选取的调查对象为 “南亚师资班” 项目的留学生, 该项目由国家汉办于 2015 年设立, 专门面向南 亚国家非中国籍人士招生, 提供奖学金资助他们到中国高校接受汉语国际教育专 业的学历教育, 毕业后回到本国从事汉语教学工作。2015 年和 2016 年在北京三 所 “南亚师资班” 项目院校就读的留学生人数为 144 人和 298 人, 分别占当年该 项目来华留学生总人数的 $38.09 \%$ 和 $38.85 \%$ 。

南亚留学生逐年增加丰富了中国大学的文化多样性和学习社群结构, 但他们 如何适应不同的学习环境, 能否应对学业上的挑战与压力, 学习动机水平与行为 表现如何, 有着怎样的学习经历和体验, 研究这些问题可为提升南亚留学生的学 习获得感, 促进中国大学改进留学生教育的教与学过程及其效果提供实证性依据。

\section{文献综述}

中国学界对来华留学生教育的研究主要集中在整体状况、教育政策、院校管理和 质量评价等宏观层面 (陈丽 \& 艾孜买提, 2016; 方宝 \& 武毅英, 2016; 郭秀晶 \& 王雯霞, 2008; 哈巍 \& 陈东阳, 2019; 韩丽丽, 2017; 刘宝存 \& 张继桥, 2018; 王军, 2006; 王祖亮, 2013）。近几年有学者开始关注来华留学生学习与发展的微观课题, 例如课堂教学、师生互动、学业评价和学习收获等 (Ma \& Wen, 2018; 文雯, 陈丽, 陈强, \& 吴运新, 2014), 但以访谈或个案定性研究方法为主, 还缺乏适用于中国 大学学习环境的相关测量工具和定量研究。同时, 来华留学生这个群体常常是跨 文化适应研究的对象, 但圊于传统跨文化适应的概念框架和理论体系, 仍然局限 于心理适应和社会文化适应的讨论, 虽然提出了学习适应的问题, 但与移民、旅 居者等群体的适应性研究结论相似 (朱国辉, 2011; 朱国辉等, 2013; 杨军红, 2009）。因此, 本文聚焦来华留学生的学习领域, 尝试突破跨文化适应研究和宏 观分析的视角, 以学习性投入理论为基础, 采用来华留学生自陈式调查工具来呈 现并分析他们在中国大学学习的表现、结果及其作用机制。

“学习性投入” 的概念被引入中国, 源于清华大学 “中国大学生学习性投入 调查问卷” (NSSE-China) 研究项目 (蒋华林, 李华, 吴芳, \& 王平, 2010; 史静詈 等, 2011; 涂冬波, 史静嬛, \& 郭芳芳, 2013)。也有人将对应的英文 “student engagement” 译为 “学习投入度” (陈静 \& 张其敏, 2014; 汪雅霜, 2013, 2015）、

“学生参与度” (丁思丹 \& 屈廖健, 2018; 侯志军 \& 张巧梅, 2012; 刘珊珊 \& 吕林 海, 2015; 吕林海, 2016, 2018; 朱红, 2010）。学习性投入最早强调的是学生个体投 入到学习活动中的时间和精力, 但随着对大学生学习与发展研究的逐步深入, 它 的内涵既包括体现学生个体在自己学业和课堂内外的有效教育活动中投入的时间 及精力, 又包括学校对学生学习提供的支持（Kahu 2013; Kahu \& Nelson, 2018）。 中国学者对西方大学生学习性投入的概念、理论和研究路径进行了持续而深入的 分析, 指出存在 “心理视角” 和 “行为视角” 两种不同模式 (史静寰 \& 王文, 2018）。基于心理视角的学习性投入概念, 着重探索其在个体心理层面的含义和 
结构, 以 Fredricks, Blumenfeld, \& Paris (2004) 提出的行为、情感和认知投入三 维结构理论为代表。基于行为视角的学习性投入概念, 强调学生和学校两方面的 投入及其相互作用（Astin, 1997; Kuh, 2009; Pace, 1984）。诚然, 学习是个体与 环境之间彼此互动的系统化过程, 但对于来华留学生学习领域的研究而言, 首先 需厘清本土化语境下来华留学生学习的表征及过程。在这种意义上，从心理视角 阐释学习性投入可能更适合于探索和解释留学生个体层面的学习投入机制，因为 外部因素如学习环境、日常生活、社会文化、人际交往等最终都是通过与留学生 的互动并经过他们内化后才会反映到他们的学习结果上来。此外, 正如某些学者 所指出的，从情感、行为和认知三维结构界定学习性投入存在 “定义模糊不清” 、

“各个维度交叉与重叠” 等问题。本研究把学习者个体的学习视为一个过程, 它 始于学习动机、终于学业成就, 而处于二者中间的是学习行为, 其中学习策略在 整个学习过程中起中介调节作用，学习策略恰当则会提升学习效率并促成学业成 就，而学习策略失误则会导致学习低效进而降低学业成就。同时，学业成就的高 低反过来又会对学习者的学习动机、学习行为以及学习策略产生影响。

\section{研究过程}

\section{研究设计}

本研究采取定量方法，设计了南亚国家来华留学生个体学习性投入调查问卷， 经过前期小规模试测和目的性抽样访谈后对调查问卷进行了修订，随后对 “南亚 师资班” 项目中在京就读的留学生正式实施问卷调查，收集数据并进行定量分析。 研究主要围绕如下两个问题展开:

1. 设计的南亚国家来华留学生个体学习性投入调查问卷是否具有良好的 信度和效度?

2. 南亚国家来华留学生个体学习性投入主要包括哪些维度? 它们与其学 业成就之间的关系如何?

\section{调查对象}

在 “南亚师资班” 项目中就读的留学生在生源地分布、语言文化、社会习俗 等背景方面存在的差异较小，这有利于本研究尽可能降低个人背景、语言和跨文 化适应等因素的影响，客观地分析个体学习性投入调查工具的结构和信效度，以 及留学生个体学习性投入状况。本研究有效调查样本为 215 人, 其中北京语言大 学 134 人, 北京师范大学 41 人, 北京外国语大学 18 人, 占在该项目北京高校就 读留学生总人数的 $65 \%$ 以上。学生主要来自巴基斯坦、尼泊尔、孟加拉国、斯里 兰卡和马尔代夫; 性别未做详细统计，但该项目招收的学生男女比例总体上呈均 衡状态; 参与调查的留学生平均年龄为 24 岁。他们在中国大学学习汉语国际教 育专业，课程以汉语和中国文化类为主，来华时间从一年至四年不等，其中绝大 多数人的汉语水平达到 HSK 五级水平以上，整体上呈现良好的跨文化适应性。

\section{测量工具}

在设计调查问卷时，重点参考了前文所述的部分理论及其测量工具，包括 Pace（1980，1984）的 “努力质量”、Fredricks 等人（2004）的学习性投入三维 
度结构、Astin（1997）的 “学习参与”、清华大学的 NESS-China 以及朱国辉 （2011）的跨文化适应调查问卷中的 “学习适应” 内容。除学生背景信息外, 问 卷共设 43 个题项, 对每个题目的回答按频率分为 “总是”、“经常”、“有 时”、“从未” 四类，并以分别赋值 4、3、2、1 分，反向题目则相反赋分。

\section{数据收集}

问卷调查从 2018 年 11 月初开始, 在征得三所院校留学生管理部门同意和协 助下, 采取分院校、限时段的网络在线调查方式 (问卷星) 进行调查和数据收集。 回收调查问卷 215 份, 剔除 IP 地址无效、填写时长过短以及缺失值较多的问卷 后, 最终获得有效样本 193 份, 有效率 $89.8 \%$ 。所有数据应用统计软件 SPSS 24.0 进行统计分析。

\section{研究结果}

\section{信度检验}

由表 1 可知, 除学习性投入的学习行为维度的信度 ( $\alpha$ 系数) 为 0.656 外, 其他各维度和问卷的整体信度 ( $\alpha$ 系数) 均大于 0.700 , 说明问卷的信度是可以接 受的。

表 1. 学习性投入调查工具信度分析

\begin{tabular}{lccc}
\hline 维度 & 信度 $(\alpha$ 系数 $)$ & 基于标准化项的 $\alpha$ 系数 & 项数 \\
\hline 学习动机 & 0.744 & .751 & 10 \\
学习行为 & 0.656 & .689 & 11 \\
学习方法 (策略) & 0.828 & .832 & 12 \\
学习结果 & 0.852 & .854 & 10 \\
\hline 总体 & 0.900 & .907 & 43 \\
\hline
\end{tabular}

\section{因素分析}

首先对学习性投入四个维度分别进行 KMO 和 Bartlett 球形检验, 结果见表 2 。 四个维度的 $\mathrm{KMO}$ 值均高于 0.700 , 说明每个维度中变量间的相关性较强, 适合做 因子分析。

\section{表 2. 学习性投入调查工具各维度的 KMO 和 Bartlett 检验}

\begin{tabular}{ccccc}
\hline \multirow{2}{*}{ 维度 } & 取样足够度的 & \multicolumn{3}{c}{ Bartlett 的球形度检验 } \\
\cline { 3 - 5 } & Kaiser-Meyer-Olkin 度量 & 近似卡方 & df & Sig. \\
\hline 学习动机 & .727 & 381.825 & 45 & .000 \\
学习行为 & .768 & 443.662 & 55 & .000 \\
学习策略 & .824 & 715.725 & 66 & .000 \\
学习结果 & .843 & 654.114 & 45 & .000 \\
\hline
\end{tabular}

表 3 列出了对学习性投入进行主成分因素分析的结果。因素分析采用具有 Kaiser 标准化的正交旋转法, 经迭代收玫后得出检验结果, 删除各维度中因子负 
荷低于 0.500 的题项, 即问卷中 Q21、Q33、Q35、Q43, 保留各维度中因子负荷 高于 0.500 的题项作为该维度所包含的题项, 并逐一进行分析并命名。

表 3. 学习性投入因子分析结果

\begin{tabular}{|c|c|c|c|c|c|}
\hline 维度 & 因子 & 题项 & & 因子负荷 & \\
\hline \multirow{8}{*}{ 学习动机 } & \multirow{4}{*}{ 融入型动机 } & Q30 & 0.691 & & \\
\hline & & Q31 & 0.672 & & \\
\hline & & Q32 & 0.671 & & \\
\hline & & Q34 & 0.717 & & \\
\hline & \multirow{4}{*}{ 工具型动机 } & Q50 & & 0.635 & \\
\hline & & Q51 & & 0.750 & \\
\hline & & Q52 & & 0.649 & \\
\hline & & Q53 & & 0.770 & \\
\hline \multirow{10}{*}{ 学习行为 } & 主动学习实践 & Q12 & & & 0.882 \\
\hline & \multirow{6}{*}{ 积极参与课堂 } & Q11 & 0.645 & & \\
\hline & & Q13 & 0.721 & & \\
\hline & & Q14 & 0.666 & & \\
\hline & & Q15 & 0.740 & & \\
\hline & & Q16 & 0.579 & & \\
\hline & & Q17 & 0.696 & & \\
\hline & \multirow{3}{*}{ 遵守学习规则 } & Q18 & & 0.579 & \\
\hline & & Q19 & & 0.731 & \\
\hline & & Q20 & & 0.524 & \\
\hline \multirow{12}{*}{ 学习策略 } & \multirow{4}{*}{ 深层学习 } & Q22 & & 0.654 & \\
\hline & & Q23 & & 0.52 & \\
\hline & & Q24 & & 0.773 & \\
\hline & & Q25 & & 0.739 & \\
\hline & \multirow{4}{*}{ 批判性学习 } & Q26 & & & 0.568 \\
\hline & & Q27 & & & 0.536 \\
\hline & & Q28 & & & 0.807 \\
\hline & & Q29 & & & 0.759 \\
\hline & \multirow{4}{*}{ 表层学习 } & Q36 & 0.735 & & \\
\hline & & Q37 & 0.740 & & \\
\hline & & Q38 & 0.808 & & \\
\hline & & Q39 & 0.796 & & \\
\hline \multirow{9}{*}{ 学业成就 } & \multirow{3}{*}{ 非认知能力 } & Q40 & & 0.800 & \\
\hline & & Q41 & & 0.775 & \\
\hline & & $\mathrm{Q} 42$ & & 0.779 & \\
\hline & \multirow{6}{*}{ 认知能力 } & Q44 & 0.632 & & \\
\hline & & Q45 & 0.652 & & \\
\hline & & Q46 & 0.655 & & \\
\hline & & Q47 & 0.701 & & \\
\hline & & Q48 & 0.760 & & \\
\hline & & Q49 & 0.683 & & \\
\hline
\end{tabular}

注. 表中的因子负荷系数是对每个维度进行因子分析的结果。 
由表 3 可见, 在学习动机维度, 对应的题项包括 “毕业后找一份满意的工 作”、“能够拿到中国大学的文凭”、“在中国学习期间可以旅游”、“满足父 母或其他人的期望” 等常见的动机类型。根据学习动机理论, 可分为工具型动机 与融入型动机两个因子, 二者的主要区别在于是否直接促发学习行为且影响持久 而深刻。对留学生而言, 对汉语和中国文化感兴趣并愿意深入学习属于融入型动 机, 而为了在中国学习期间旅游、满足父母或重要他人的期待、毕业后找到一份 满意的工作等则属于工具型动机。

在学习行为维度, 对应的题项包括 “课堂上专心致志地听讲”、“课堂上提 问或主动回答问题”、“积极参与小组课堂讨论”、“按时完成作业” 等外显性 的活动与表现。提取的三个因子分别命名为 “遵守学习规则”、“积极参与课堂” 和 “主动学习实践”。因为考勤制度和奖学金标准属于刚性规定, 如有违反则可 能通不过考试或被取消奖学金, 因此留学生在很大程度上是被动地遵守; 在课堂 上与老师和同学进行互动讨论、小组合作学习则是一种主动、积极参与学习的表 现和态度; 此外, 留学生在课外主动与其他学生尤其是中国人交流, 是他们学习 汉语与了解中国文化非常重要而快捷的途径, 但往往因人而异, 性格活泼、外向 的留学生倾向于主动寻求语言实践的机会和资源, 从而在语言交际能力和跨文化 理解方面表现得更加优秀。

在学习策略维度, 对应的题项包括 “在思考问题时能够从不同学科中汲取知 识来组织资料”、“能将自己课堂上学到的知识联想到现实生活中存在的社会现 象或问题”、“通过课堂上的学习能够意识并检验自己思维的优缺点”、“能够 尝试从他人的角度思考他人的问题” , 以及 “在学习中是否需要借助母语或英语 来理解知识” 等。提取的三个因子分别命名为 “表层学习”、“深层学习” 和 “批判性学习”。记忆、模拟分析和信息使用等是表层学习的重要方法, 它以完 成学业考核为目标, 尤其是知识点识记、理解和分析为表征; 深层学习主要体现 为努力掌握汉语思维方式、利用跨学科知识整合学习材料、联系并思考社会生活 现象与问题, 表现知识掌握的深刻性和实践运用的灵活性; 而批判性学习的特征 是在学习过程中有意识地检视自己的思维方式、尝试从他人立场思考他人观点、 自我评判过往经历及观念变化等, 即学习者能够运用所学知识实现质的转变, 掌 握辨识、判断和创新等更高阶的学习能力。

在学业成就维度，对应的题项包括 “帮助我掌握了汉语的基本知识”、“了 解中国文化”、“在日常生活中与其他留学生用汉语交流”、“拓宽了我的知识 面和视野”、“提高了我与他人相处和团队合作能力”、“锻炼了我的逻辑性思 维”、“增长了我解决问题的信心和能力”、“鼓励我尊重和理解不同的观点”

等知识和技能。标准化成绩固然是衡量的重要标准但绝非唯一, 通过学习者个人 的体认来反映他们如何看待与评价自己取得的学业成也是重要的考量因素, 即学 习者依据自身主观经验而产生的学习获得感。依据因子分析的结果, 可区分为认 知能力与非认知能力两类 (有的也称智力因素与非智力因素), 前者主要与汉语 知识的学习及运用能力有关, 后者则体现为团队合作、逻辑思维、自我效能感、 多元文化理解以及全球化胜任力等综合素养。

\section{相关性分析}

表 4 列出了学习动机、行为、策略与学业成就之间的相关分析结果。除主动 学习实践与认知能力不存在显著相关外, 其他各因子与学业成就总体及其认知能 
力与非认知能力维度均呈显著的正相关关系。与学业成就总体相关性较强的因子 由高到低排序为融入型学习动机、表层学习策略、积极参与课堂行为等。与认知 能力相关性较强的因子依次为遵守学习规则行为、批判性学习策略、融入型学习 动机、表层学习策略和积极参与课堂行为等。与非认知能力相关性较强的因子依 次为融入型学习动机、积极参与课堂行为、表层学习策略等。

\section{表 4. 学习动机、行为、策略与学业成就皮尔逊相关分析}

\begin{tabular}{|c|c|c|c|c|}
\hline \multirow{2}{*}{ 维度 } & \multirow{2}{*}{ 因子 } & \multicolumn{3}{|c|}{ 学业成就 } \\
\hline & & 认知能力 & 非认知能力 & 总体 \\
\hline \multirow{2}{*}{ 学习动机 } & 融入型动机 & $0.491 * * *$ & $0.470 * * *$ & $0.545 * * *$ \\
\hline & 工具型动机 & $0.274 * * *$ & $0.287 * * *$ & $0.321 * * *$ \\
\hline \multirow{3}{*}{ 学习行为 } & 遵守学习规则 & $0.520 * * *$ & $0.299 * * *$ & $0.348^{* * *}$ \\
\hline & 积极参与课堂 & $0.429 * * *$ & $0.430 * * *$ & $0.490 * * *$ \\
\hline & 主动学习实践 & 0.128 & $0.149 *$ & $0.160 *$ \\
\hline \multirow{3}{*}{ 学习策略 } & 表层学习 & $0.485 * * *$ & $0.421 * * *$ & $0.510 * * *$ \\
\hline & 深层学习 & $0.268 * * *$ & $0.239 * * *$ & $0.286 * * *$ \\
\hline & 批判性学习 & $0.520 * * *$ & $0.385 * * *$ & $0.423 * * *$ \\
\hline
\end{tabular}

$* P<0.05 ; * * * P<0.001$ (双侧检验)。

\section{结论与讨论}

第一，此次调查采用的测量工具即 “南亚国家来华留学生个体学习性投入调查问 卷” 经检验具有良好的信效度, 包括四个维度即学习动机、学习行为、学习策略 与学业成就, 在各维度下共提取出了 10 个因子。由于在 “南亚师资班” 项目学 校学习的来华留学生内部差异较小, 在样本结构复杂的留学生群体, 如生源地多 样、学历层次多样、社会文化背景多元的群体使用该量表时, 有必要将人口统计 学信息、学科、专业和院校特征等纳入考虑范围, 进一步验证调查工具的信效度。

第二, 从各维度因子与学业成就的相关性看, 融入型学习动机与学业成就的 相关性更大, 说明对汉语和中国文化的兴趣是促进留学生学习性投入的持久且稳 定的内部动机, 应当作为选拔或审核来华留学生的重要标准加以精准判断。这一 点对招收来华留学的学历生尤为重要, 因为学历生通常学习周期长、任务重、压 力大, 只有学习者内心对汉语和中国文化保持强烈的积极感知和学习兴趣, 才可 能降低出现学习倦急或 “混日子” 心态的可能性。对于教师而言, 应该将激发留 学生的融入型学习动机纳入教学目标和教学环节之中, 关注不同学习阶段、不同 环境下留学生学习动机的动态变化及强弱水平, 合理地选择教学内容与材料, 采 取多样化、趣味性的教学方法和恰当的教学策略。学校也应该通过丰富的有教育 意义的文化体验活动, 增进留学生对汉语与中国文化的积极感知。

第三, 根据南亚国家来华留学生的自我认知与评价得知, 表层学习、积极参 与课堂和学业成就各个维度的相关性均较强, 这从某种程度上说明他们更倾向于 将学业成就归因于记忆背诵和在课堂上积极表现等表层行为, 也反映出目前中国 大学对来华留学生的学习考核和评价仍然是以应试和教师主导为特征, 对理论联 
系实践、自主性学习和批判性分析等高阶思维能力的培养尚未得到足够重视与积 极实践。中国高校对来华留学生的教育培养应符合大学生学习发展的阶段性特征 与一般性规律, 转变以教师为中心、灌输式的教学模式, 以培养知识获取、批判 性思维和学术研究能力为重点, 进一步强化留学生的深层学习和批判性学习, 为 来华留学生未来终身学习和个人发展提供充分的支持。

总体而言, 南亚国家来华留学生的汉语水平、应用能力以及专业知识等认知 能力都得到较大提升, 同时他们也意识到并且能够正面评价自身在全球视野、团 队合作、跨文化理解等非认知能力方面的发展与变化, 基本能够适应中国大学的 学习并获得较积极的学习体验和收获。从调查结果看, 学习性投入对留学生的学 业成就具有较强的解释力。这就要求中国大学及教师更充分有效地利用课堂教学、 教育资源和校园文化增强师生、生生互动, 提升来华留学生的个体学习性投入水 平, 进而提高学校留学生的培养质量。

\section{参考文献}

Astin, A. W. (1997). What matters in college?. New York, NY, US: Jossey-Bass.

Fredricks, J. A., Blumenfeld, P. C., \& Paris, A. H. (2004). School engagement: Potential of the concept, state of the evidence. Review of Educational Research, 74(1), 59109.

Kahu, E. R. (2013). Framing student engagement in higher education. Studies in higher education, 38(5), 758-773.

Kahu, E. R., \& Nelson, K. (2018). Student engagement in the educational interface: understanding the mechanisms of student success. Higher Education Research \& Development, 37(1), 58-71.

Kuh, G. D. (2009). The national survey of student engagement: Conceptual and empirical foundations. New directions for institutional research, 141(1), 5-20.

Ma, J., \& Wen, Q. (2018). Understanding international students' in-class learning experiences in Chinese higher education institutions. Higher Education Research \& Development, 37(6), 1186-1200. http://doi.org/ 10.1080/07294360.2018. 1477740

Pace, C. R. (1980). Measuring the quality of student effort. Current Issues in Higher Education, 2(1), 10-16.

Pace, C. R. (1984). Measuring the quality of college student experiences. An account of the development and use of the college student experiences questionnaire. Retrieved from https://files.eric.ed.gov/fulltext/ED255099.pdf.

陈静, \& 张其敏. (2014). 学习投入度与本科教学质量标准. 教育评论, (5), 29-31.

陈丽, \& 伊莉曼·艾孜买提. (2016). “一带一路”沿线国家来华留学教育近 10 年发展 变化与策略研究. 比较教育研究, (10), 27-36.

丁思丹, \& 屈廖健. (2018). 基于“双维度”的大学生学习参与行为研究. 西南师范大 学学报(自然科学版), 43(10), 157-164.

方宝, \& 武毅英. (2016). 高等教育来华留学生的变化趋势研究—一基于近十五年统 计数据的分析. 高等教育研究, 39(2), 23-34.

郭秀晶, \& 王雯霞. (2008). 来华留学高等教育的政策分析与制度变迁. 北京科技大 学学报(社会科学版), 24(4), 147-151. 
哈巍, \& 陈东阳. (2019). 人才流动与教育红利一一来华留学教育研究综述. 教育学 术月刊, (3), 55-64.

韩丽丽. (2017). 如何提升来华留学教育的竞争力一一基于规模总量和学历结构视 角的经验分析. 北京师范大学学报 (社会科学版), 5, 18-30.

侯志军, \& 张巧梅. (2012). “学生学习参与” 研究的发展阶段及价值分析. 煤炭高等 教育, 30(1), 1-4.

蒋华林, 李华, 吴芳, \& 王平. (2010). 学习性投入调查: 本科教育质量保障的新视角. 高教发展与评估, 26(4), 45-53.

刘宝存, \& 张继桥. (2017). “一带一路”沿线国家来华留学学历教育：地位、问题与 对策. 北京教育: 高教版, (5), 11-14.

刘珊珊, \& 吕林海. (2015). 本科生学习参与对其能力发展的影响的实证研究——基 于南京大学 SERU 问卷调查. 教学研究, 38(5), 1-5.

吕林海. (2016). 大学生学习参与的理论缘起, 概念延展及测量方法争议. 教育发展 研究, 36(21), 70-77.

吕林海. (2018). 全球视野下中国一流大学本科生的学习参与: 当前表现与努力方 向一一基于中、美、英、日 SERU 调查数据(2017-2018)之解析. 教学研究, 41(6), 7-11.

马佳妮. (2018). 来华留学生就读感知形成路径及积极感知提升策略. 中国高教研究, (2), 37-41.

史静寰, \& 王文. (2018). 以学为本, 提高质量, 内涵发展: 中国大学生学情研究的学 术涵义与政策价值. 华东师范大学学报( 教育科学版), (4), 18-27,162.

史静寰, 涂冬波, 王纾, 吕宗伟, 谢梦, \& 赵琳. (2011). 基于学习过程的本科教育学情 调查报告 2009. 清华大学教育研究, 32(4), 9-23.

涂冬波, 史静睘, \& 郭芳芳. (2013). 中国大学生学习性投入调查问卷的测量学研究. 复旦教育论坛, $11(1), 55-62$.

汪雅霜. (2013). 大学生学习投入度的实证研究——基于 2012 年“国家大学生学习 情况调查. 中国高教研究, (1), 32-36.

汪雅霜. (2015). 大学生学习投入度对学习收获影响的实证研究一一基于多层线性 模型的分析结果. 国家教育行政学院学报, (7), 76-81.

王军. (2006). 来华留学研究生教育现状分析. 中国高教研究, (6), 21-23.

王祖亮. (2013). 来华留学生教育发展变化、动因分析及未来展望一一基于 20042011 年统计数据的实证分析. 大学(学术版), (12), 47-54,.

文雯, 陈丽, 陈强, \& 吴运新. (2014). 课堂学习环境与来华留学生学习收获的研究一 一以清华大学为例. 清华大学教育研究, 35(2), 107-113.

杨军红. (2009). 来华留学生跨文化适应问题研究. 上海社会科学院出版社.

朱国辉. (2011). 高校来华留学生跨文化适应问题研究（博士学位论文）.上海华东 师范大学, 上海.

朱国辉, 谢安邦, \& 许美德. (2013). 高校来华留学生跨文化适应问题研究. 高等教育 研究, 34(9), 94.

朱红. (2010). 高校学生参与度及其成长的影响机制一一十年首都大学生发展数据 分析. 清华大学教育研究, 6, 40-48, 68 . 
中华人民共和国教育部。(2019)。2018 年来华留学统计. http://www.moe.gov.cn/jyb_xwfb/gzdt_gzdt/s5987/201904/t20190412_377692.h tml

\section{作者简介（Author biography）}

余蓝 (通讯作者), 北京语言大学汉语教育学院副教授, 研究兴趣主要包括来华 留学生学习参与、跨文化适应、教育测量与评价、国际学生流动等。电子邮箱: yulan@blcu.edu.cn

LAN YU (corresponding author) currently works in the Faculty of Humanities and Social Science at Beijing Language and Culture University, China. Lan Yu conducts research in the fields of student engagement, cross-cultural adaptation, educational measurement and evaluation, and international student mobility. Email: yulan@blcu.edu.cn

朱述承, 北京语言大学信息科学学院硕士研究生, 研究兴趣和方向为计算语言学、 教育测量与评价。电子邮箱: zhu_shucheng@126.com

SHUCHENG ZHU is a postgraduate student in the School of Information Science at Beijing Language and Culture University, China. His research interests include computational linguistics, educational measurement and evaluation. Email: zhu_shucheng@126.com 